Convention between the United States of America and the United Mexican States for the solution of the problem of the Chamizal, The Department of State Bulletin 1963, pp. $4^{81-4} 8_{2}\left(8_{3}\right)$.

Convenzione sulle relazioni consolari, R.D.I. 1963, pp. 504-528 (1 29).

Declaration conjointe sur la limite extérieure du Rio de la Plata signée à Montevideo le 30 janvier 196i, R.G.D.I.P. I963, pp. 722-723 (83).

DocumENTs (Basic) including amendments approved by the fifteenth World health assembly, May 1962, World Health Organization, December 1962, 194 pp. (Also in French) (157).

Dokumentation der europäischen Integration mit besonderer Berücksichtigung des Verhältnisses EWG-EFTA, Bonn 196I, 474 pp. (I 70a, I 40).

DOKumENTEN betreffende de verdragen en overeenkomsten op 8 april ig6o tussen de Nederlandse en West-Duitse regering gesloten, I.S. 1963, pp. I $5^{1-}$ 208 (455).

EXtradition AGT, I.J.I.L. i962, pp. 531-544 (252).

Fernmeldevertrag (Internationaler, Genf 1959). Schlussprotokoll, Zusatzprotokolle, Entschliessungen, Empfehlungen und Begehren, Vienna 196 I, i 7o, 178 pp. (153).

GatT (Das), Eine Textausgabe des Allgemeinen Zoll-und Handelsabkommens begleitet von einigen für die Anwendung des GATT wichtigen Protokollen, F. K. Liebich, Baden-Baden-Bonn 196r, 137 pp. (140).

Die Gippenkonferenz der unabhängigen Staaten Afrikas in Addis Abbeba in Mai 1963, E.A. 1963, D 313- D324 (52).

Materials (International legal). Current documents I-, Washington, D.C. $1962(22,24 \mathrm{a})$.

Terzo Protocollo addizionale all'Acordo generale sui privilegi e le immunità del Consiglio d'Europa, con annesso statuto del fondo di ristabilimento, R.D.I. 1963, pp. 286-3 I 3 ( I 70a).

Schlussakte der Konferenz der Vereinten Nationen über den diplomatischen Verkehr und die diplomatischen Immunitäten, I.R.D. 1961, pp. 8 I-132 (1 25).

UrKunden der Algerien-Konferenz zu Evian vom 18. März 1962 (In French), A.V. 1963, pp. 21 2-238 $(455,352)$.

VerTRAGSWERK zur Gründung einer Europäischen Weltraumforschungs-Organisation vom 14. Juni 1962, A.V. 1963, pp. 196-2 1 1 (1 48a).

\title{
II. Private International Law
}

Conventron on the liability of operators of nuclear ships, A.J.I.L. 1963, pp. 268$278(307)$.

Property Commission (The Anglo-Japanese). Decisions, London 196 I, i 45 pp. $(204,497 \mathrm{~d})$.

\section{ERRATUM}

In the note on page $389-390$ of volume $X$ (Netherlands Judicial Decisions) the letters $a, b, c$ and $d$ should be read as $c, d$, e and $f$. 